\title{
DEVELOPMENT OF TEXTURE IN AN ELC-BH SHEET WITH VERY HIGH R-VALUE DURING ANNEALING
}

\author{
XIAOJUN GUAN*, XIAOJUN HU, QIULIN WU and KE YU** \\ *Department of Metal Forming, University of Science and Technology \\ Beijing, P. R. China, **Shougang Metallurgical Research \\ Institute, Beijing, P. R. China
}

(Received 5 May 1995)

\begin{abstract}
The texture change in an ELC-BH sheet steel with very high $\bar{r}$ value produced through a new technology invented in our laboratory during annealing has been successfully investigated by means of the methods of ODF analysis and metallographic structure observation in this paper. Thus, the developmental law of the texture which consists of even stronger $\{111\}$ texture with main $\{111\}<110>$ components as compared with that of texture in conventional technology during annealing has been discovered. The experimental results are described as follows: 1) It is different from conventional technology that the velocity of both nucleation and growth to the recrystallization grains of $\{111\}<110>$ orientations is always larger than that of $\{111\}<112>$ orientations. 2) The cause why this law is engendered may be related to that the advantage, that cold deforming stored energy of $\{111\}<110>$ orientation grains is higher than that of $\{111\}<112>$ orientation grains, is brought into fully play in the cold rolled sheet with stronger $\{111\}$ texture during annealing.
\end{abstract}

KEY WORDS: Texture, recrystallization annealing, extra low-carbon, high strength, bake-hardening.

\section{INTRODUCTION}

Extra low-carbon sheet steel products represented by interstitial-free steel (i.e. IF sheet) have been widely used in automotive industry today. Extra low-carbon and high strength bake hardening sheet steel (i.e. ELC-BH sheet), which is one of these products, has been warmly welcome by users because of its excellent combined properties which consist of extra deep-drawing property, high strength and anti-dent ability. According to conventional production technology, $\bar{r}$-value about 2.0 or so can be usually gained in the sheet and can hardly be improved again because of adding microalloying element phosphorus into the steel. With the rapid development of automobile industry, the demands on the properties of drawing sheet steels are changing into two ways. One way, concernes the development of some new drawing technologies to simplify the press process and to prevent weld corrosion in order to produce more beautiful streamline of autobody, such as "one-forming" or "body-in-whole forming". Hence, the drawing properties should be better and better. Another way, concernes energy saving and weight reduction. Higher strength and smaller thickness sheet steels will be required. Clearly, the properties of EIC-BH sheet produced by conventional processing will not satisfy the development demands of automobile industry. Hence, we developed a new 
technology by which an $\bar{r}$ value as high as 2.67 and other good properties was produced in our laboratory. The character of this new technology was adding a previous texture treatment process between conventional hot- and cold-rolling process through which a cold-rolled sheet which promoted the development of $\{111\}$ annealing texture could be obtained (Guan et al. 1995). Our experimental results showed that the annealing texture gained by the new technology consists of even stronger $\{111\}$ texture as compared with that by conventional technology, and the crystal orientations corresponding to the peak values of orientation density had also been changed from conventional $\{111\}<112>$ orientations to $\{111\}<110>$ orientation (Guan et al. 1995). In order to reveal further the cause why these changes took place, we carefully investigate texture development during annealing in the new and the conventional technology respectively.

\section{EXPERIMENTAL MATERIALS AND METHODS}

An ELC-BH steel melted in our laboratory was selected for experimental investigation. The hot-rolled sheets obtained after hot rolling and coiling were divided into two groups. One group was cold rolled directly in conventional technology, while cold rolling of another group was carried out after the previous texture treatment process of the new technology. The two groups had the same cold reduction and sheet thickness.

Twenty samples which had $30 \times 25 \mathrm{~mm}^{2}$ size were respectively cut from the cold rolled sheets. Then these samples were put into the treating oven to simulate continuous annealing processing: The parameters of hot treating are shown in Figure 1. Surface hardness, metallographic structure, and texture were determined on the hot treated samples. In this paper, samples corresponding to six different states, that is cold rolled state, late stage of recovery, recrystallization stage, initial stage of grain growth, stage of grain growth and state of finished annealing, were respectively selected, based on the variance curves of surface hardness and observation of metallographic structure, in order to study the effects of the conventional and the new technology on their microstructures and textures.

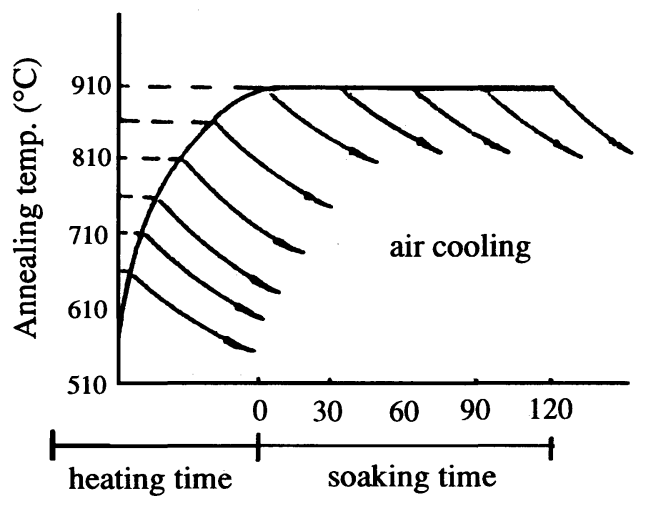

Annealing time (s)

Figure 1 The process of heating treatment. 
The surface hardness of the samples was tested on an HD9-45 type optical surface hardometer and the metallographic structure of the side plane parellel ro rolling direction was observed. The texture analysis of the samples was conducted on a Rigaku-3014 type X-ray diffractometer using $M o k_{\alpha}$ radiation at $40 \mathrm{kV}$, and $20 \mathrm{~mA}$. The testing procedure was as follows. At first, two whole pole figures of (110) and (200) were determined by the method of combining transmission with reflection. Then, the orientation distribution functions (ODFs) were calculated from the pole figures. The results are given in $\Phi=45^{\circ}$ sections.

Samples for metallographic strucutre and texture measurement were cut from the hot treated samples. The size of the microstructure samples was $25 \times 10 \mathrm{~mm}^{2}$, while the size of the texture samples was $15 \times 15 \mathrm{~mm}^{2}$. The thickness of the texture samples was $70 \mu \mathrm{m}$ and their center was at $1 / 4$ sheet thickness from the sheet surface. The texture of the sheet surface may be different, to some extent, from that of the center of the sheet. Hence, the measurements were carried out in layers of uniform thickness in order to have the same conditions in all samples.

\section{EXPERIMENTAL RESULTS}

Under the conditions of the conventional and the new technology, the changes of metallographic structure during annealing are respectively shown in Figure 2 and
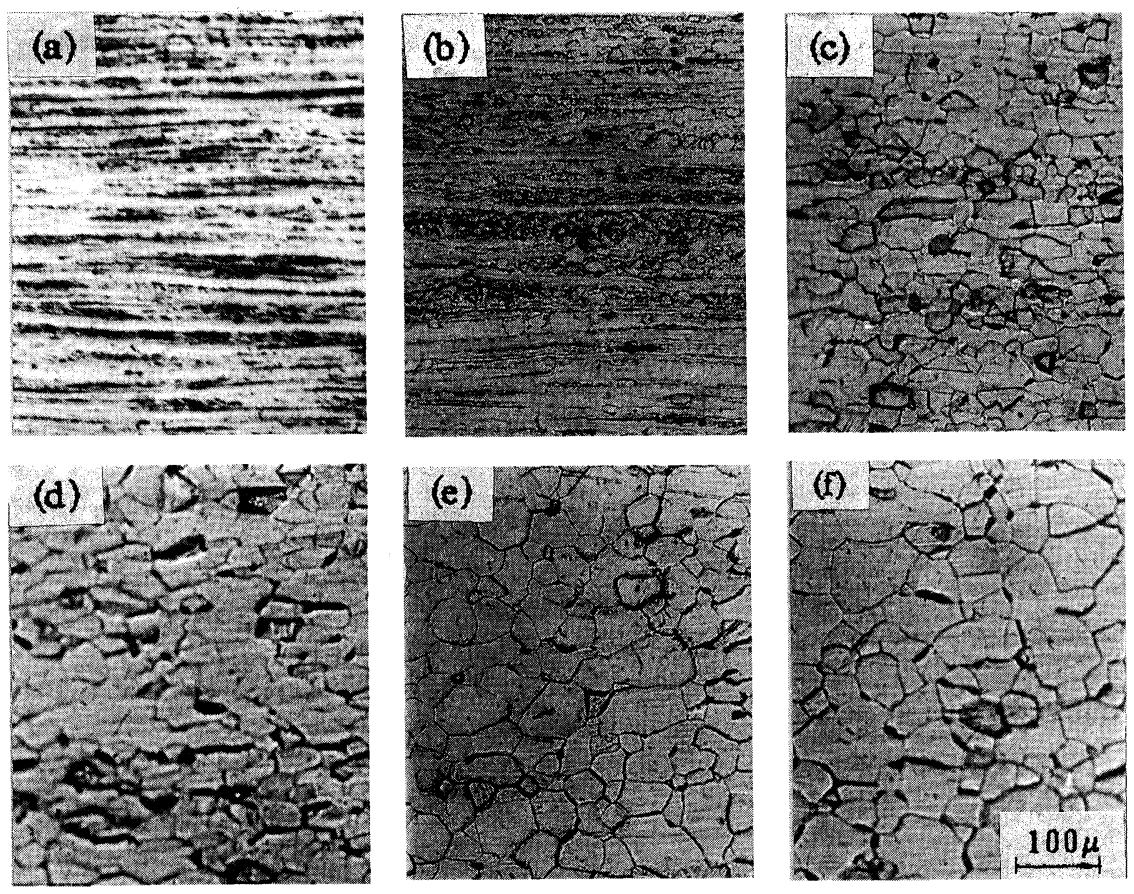

Figure 2 The changes of microstructure during annealing in cold rolled sheet produced by conventional technology a) cold rolled state b) late stage of recovery c) recrystallization stage d) initial stage of grain growth e) stage of grain growth f) state of finished annealing. 

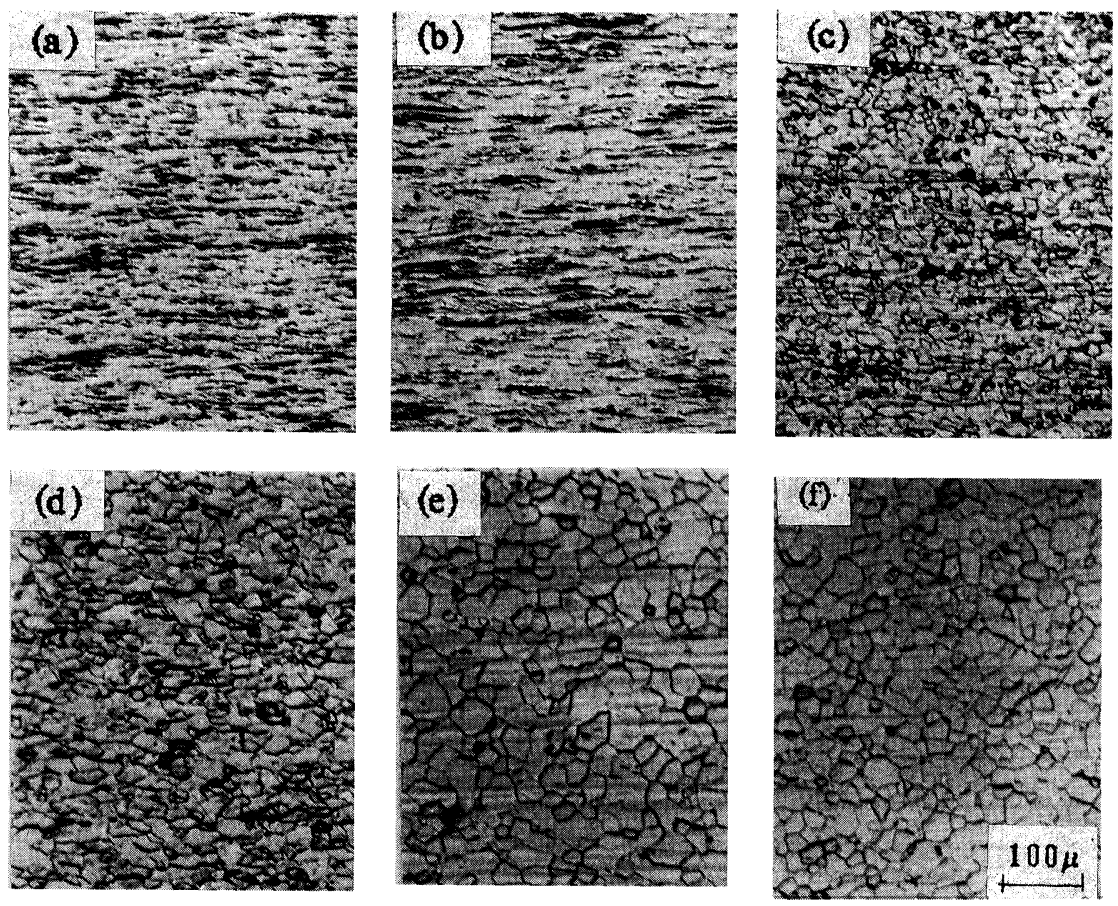

Figure 3 The changes of microstructure during annealing in cold rolled sheet produced by new technology a) cold rolled state b) late stage of recovery c) recrystallization stage d) initial stage of grain growth e) stage of grain growth $\mathrm{f}$ ) state of finished annealing.

Figure 3. It is clearly seen that the ferrite microstructures of the two cases go through the same changes from cold deformed biscuit grains to forming and growing of recrystallization nuclei until formation of equi-axed and bigger recrystallization grains, that is three continuously changing stages (recovery $\rightarrow$ recrystallization $\rightarrow$ grain growth) can be seen. The observations show that the grain sizes of the ferrite, corresponding to every annealing state in the new technology, are generally smaller than those in the conventional processing. Therefore, the new technology also increases the amount of the annealed micostructure.

The textures corresponding to the two technologies are respectively seen in Figure 4 and Figure 5. In the conventional technology, the major components of cold rolling texture $\{100\}$ and $\{h k l\}<110>$ still develop continuously in the late stage of recovery, as shown in (a) and (b) of Figure 4. This is due to a great number of sub-grains formed by ploygonization. In the recrystallization stage, as shown in (c) and (d) of Figure 4, a large number of $\{111\}$ recrystallization grains are formed by nucleation and growth. In the growth stage of the recrystallization grains, as shown in (d) to (f) of Figure 4 , the $\{111\}$ texture becomes stronger and stronger with the growth of these grains by consuming grains of other orientation whose size is smaller.

In the new technology, the change of the $\{111\}$ texture during annealing is even stronger as compared with the conventional technology. In all states corresponding to the conventional annealing procedure, the orientation density of the $\{111\}$ texture is higher and the peak orientation changes from $\{111\}<112>$ to $\{111\}<110>$ when 


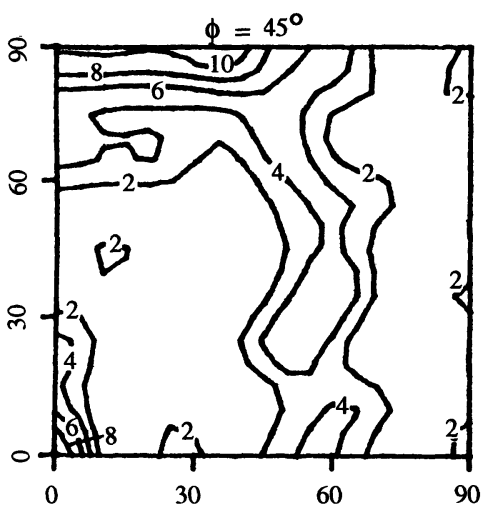

(a)

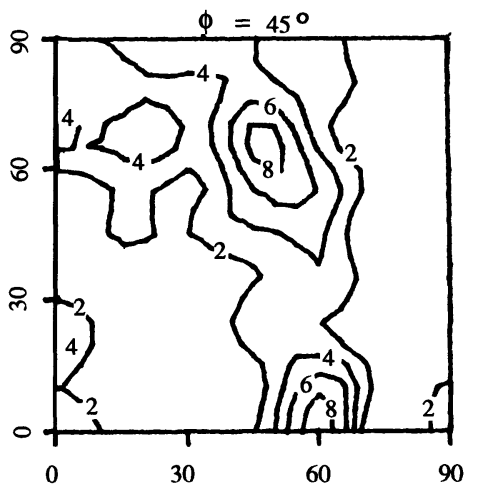

(c)

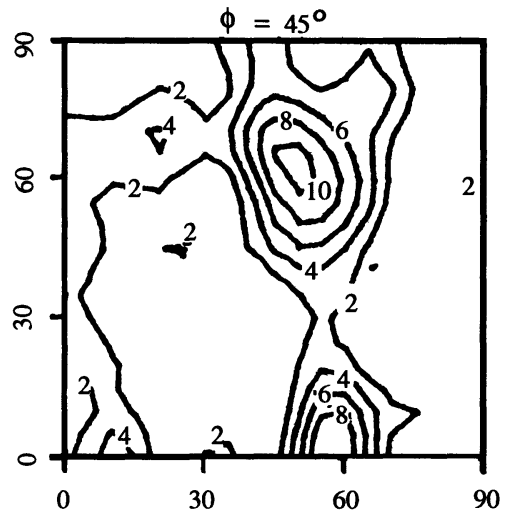

(e)

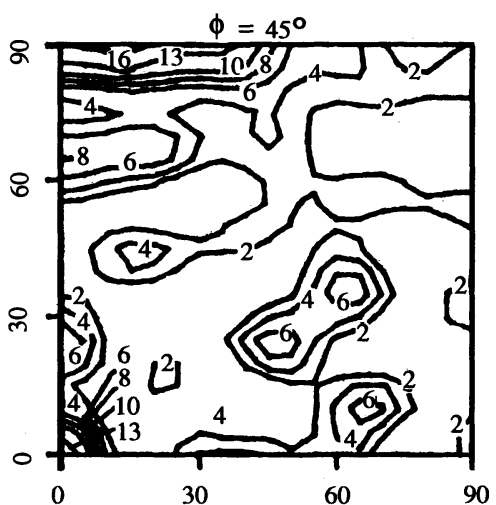

(b)

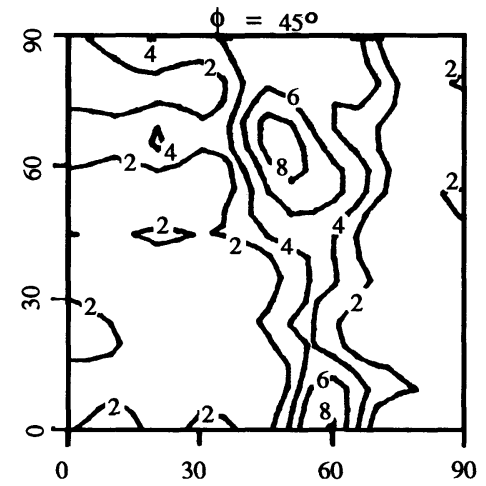

(d)

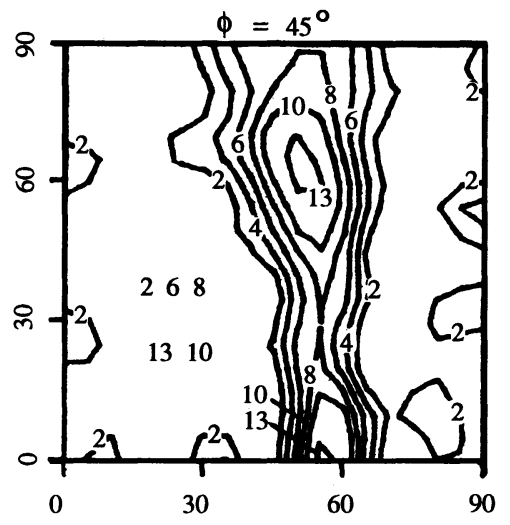

(f)

Figure 4 The changes of texture during annealing in cold rolled sheet produced by conventional technology a) cold rolled state b) late stage of recovery c) recrystallization stage d) initial stage of grain growth e) stage of grain growth f) state of finished annealing. 


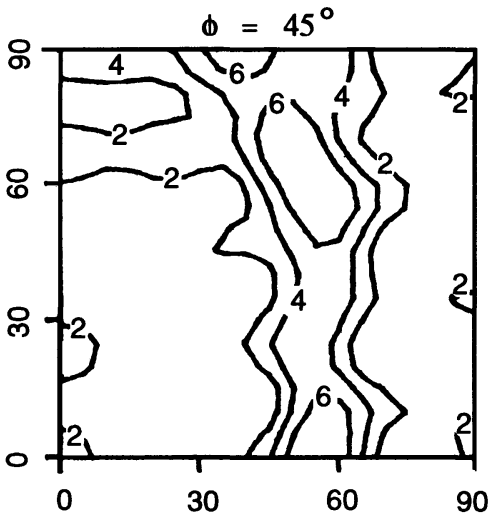

(a)

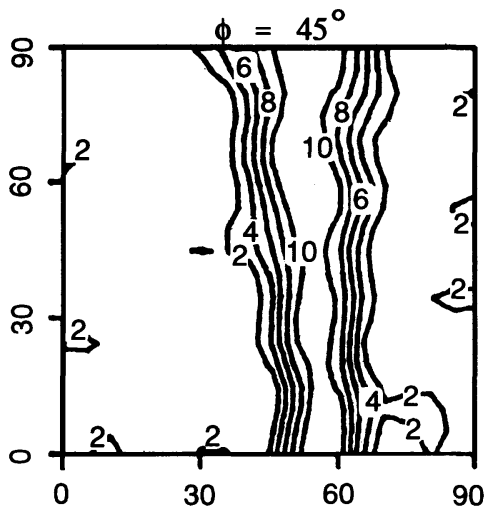

(c)

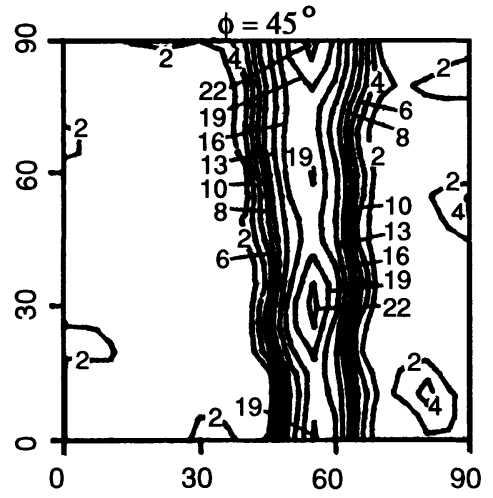

(e)

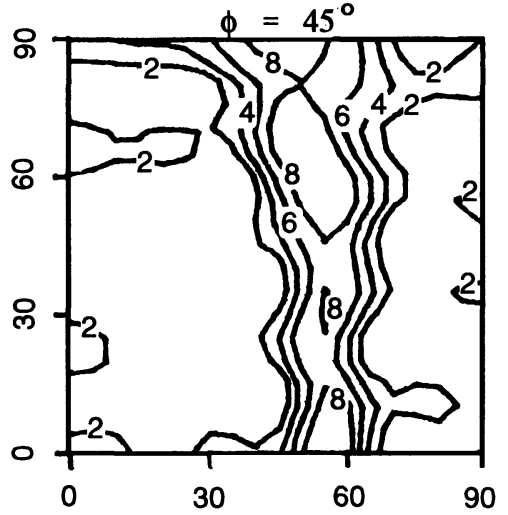

(b)

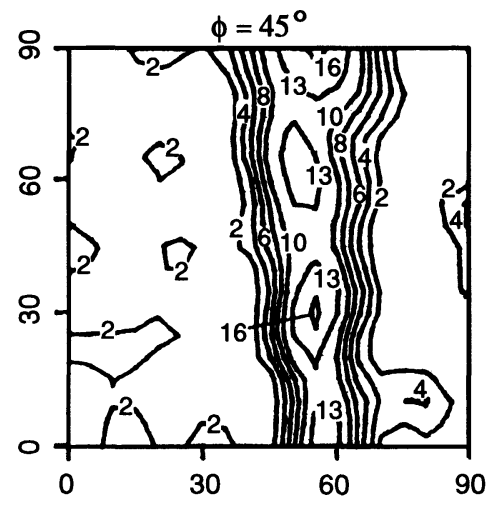

(d)

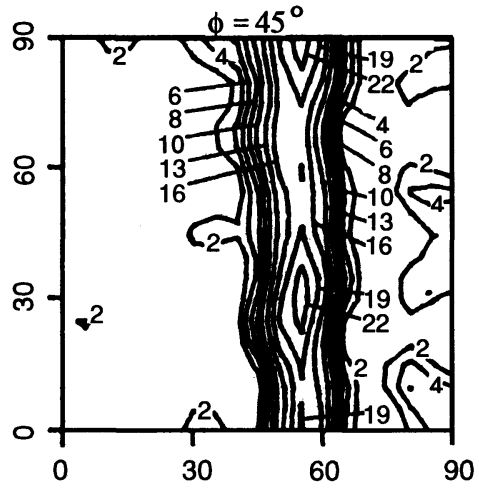

(f)

Figure 5 The changes of texture during annealing in cold rolled sheet produced by new technology a) cold rolled state b) late stage of recovery c) recrystallization stage d) initial stage of grain growth e) stage of grain growth f) state of finished annealing. 
recrystallization begins, as is shown in (c) of Figure 5. During the following process from nucleation to growth of grains which have definite size, as shown in (c) to (e) of Figure 5, the orientation densities near $\{111\}<110>$ increase faster than those near $\{111\}<112>$ so that the $\{111\}<110>$ texture components finally constitute the major part of the $\{111\}$ annealing texture. In the late stage of grain growth, as shown in (e) and (f) of Figure 5, the $\{111\}$ texture does not generally develop further, but the $\{111\}<110>$ components still increase a little and the $\{110\}$ component also develops at a certain extent. In short, during the development of recrystallization texture in the new technology, the velocity of $\{111\}$ texture development always is larger than that in the conventional technology, and the $\{111\}<110>$ texture components is stronger than the other components of the $\{111\}$ texture. This leads to the very high $\bar{r}$ value which can not be obtained by the conventional technology.

\section{DISCUSSIONS}

The difference of the development of recrystallization texture in the new technology from that in the conventional technology must be attributed to its cold rolled parent state which has stronger $\{111\}$ texture components. Because the formation of the recrystallization texture in ELC-BH sheet follows the mechanism of preferential necleation and growth of $\{111\}$ texture like that in IF steel (Guan et al. 1993), the grains of $\{111\}$ orientation in the new cold rolled sheet have not only higher orientation density, but also higher stored deformation energy. Hence, the advantage of the $\{111\}$ grains in the processes of necleation and grain growth is strengthened further, thus resulting in the development of even stronger $\{111\}$ texture.

The reason why the $\{111\}<110>$ component is stronger than $\{111\}<112>$ may be related to its higher cold deforming stored energy. As shown in (b) of Figure 5, the orientation density of $\{111\}<110>$ is close to that of $\{111\}<112>$ in the late stage of recovery though it is lower than that of $\{111\}<112>$ in the cold rolling texture. It is clearly stated that more sub-grains emerge within the $\{111\}<110>$ grains after polygonization because of their higher stored energy. The difference of the stored energy becomes the main reason for the development of the $\{111\}$ texture. Hence, nuclei of $\{111\}<110>$ orientations can form and grow prior to those of $\{111\}<112>$ orientations during recrystallization, as shown in (c) and (d) of Figure 5, so that both, quantity and size, of these grains are larger than those of $\{111\}<112>$ orientations. In the following process of grain growth, the $\{111\}<110>$ grains will continuously keep the advantage of competition with the $\{111\}<112>$ grains by consuming other smaller grains, thus leading to their even stronger development. In the late stage of grain growth, the advantage does not further exist because other smaller grains have been already consumed. Therefore, the development of the two texture components tends to saturations.

Why is the development of the $\{111\}<110>$ texture components with higher deformation stored energy weaker as compared with $\{111\}<112>$ components in conventional technology? This probably relates to higher energy of the grain boundaries between the grains of $\{111\}<112>$ and $\{100\}$ or $\{h k l\}<110>$ orientations in the cold rolled sheet, which are parallel to sheet surface. Because there are more $\{100\}$ and $\{h k l\}<110>$ grains in the conventionally cold rolled sheet, the grains of $\{111\}$ $<112>$ orientations form nuclei prior to the $\{111\}<110>$ grains on the grain boundaries which they respectively possess in common with $\{100\}$ or $\{h k l\}<110>$ 
orientations, so that both, amount and growth velocity, of these nuclei are larger than those of the $\{111\}<110>$ orientations. This is the reason why the advantage of stored energy in the grains of $\{111\}<110>$ orientations does not become the major power of texture development in the new technology, thus leading to the even stronger development of $\{111\}<112>$ components as compared with $\{111\}<110>$ components.

Hence, it is obviously understood why the even stronger $\{111\}$ texture having mainly $\{111\}<110>$ components can be developed during annealing in a cold rolled parent state which consists of stronger $\{111\}$ components.

\section{CONCLUSIONS}

1. The velocity of nucleation ad growth of the recrystallization grains corresponding to $\{111\}<110>$ orientations is always larger than that corresponding to $\{111\}$ $<112>$ orientations during the annealing in the new technology. Thus even stronger $\{111\}$ annealing texture with stronger $\{111\}<110>$ components as compared with the conventional technology is obtained.

2. The reason for the difference between the development of the $\{111\}$ annealing textures in the conventional and the new technology may be that the advantage, of stored energy of the $\{111\}<110>$ grains is higher than that of the $\{111\}$ $<112>$ grains, in the cold rolled sheet with stronger $\{111\}$ texture.

\section{References}

Guan Xiaojun, et al. (1995), Investigation on the Texture of an ELC-BH Sheet with Very High $\bar{R}$-Value Processed by New Technology, Textures and Microstructures, Vol. 23, P21-27.

Guan Xiaojun, et al. (1993), The Annealing Microstructure and Properties in Extra Low-Carbon and high Strength Bake-Hardening Sheet Steel, Journal of University of Science and Technology Beijing, Vol. 15, No. 6, P580-585. 